\title{
Advances in neutron-induced resonance reaction cross section studies at GELINA
}

\author{
A. Borella ${ }^{1}$, F. Gunsing ${ }^{2}$, S. Kopecky ${ }^{1}$, I. Ivanov ${ }^{1}$, C. Mihailescu ${ }^{1}$, M. Moxon ${ }^{3}$, A.J.M. Plompen ${ }^{1}$, C. Sage ${ }^{1}$, \\ P. Schillebeeckx ${ }^{1, a}$, P. Siegler ${ }^{1}$, and I. Sirakov ${ }^{1}$ \\ ${ }^{1}$ EC-JRC-IRMM, 2440 Geel, Belgium \\ ${ }^{2}$ CEA/Saclay, DSM/DAPNIA/SPhN, 91911 Gif-sur-Yvette, France \\ ${ }^{3}$ Hyde Copse 3, Marcham, UK
}

\begin{abstract}
The neutron time-of-flight facility GELINA at the IRMM Geel, Belgium has been especially designed for neutron-induced reaction cross section studies in the resonance region. It is a multi-user facility, serving up to 10 different experiments simultaneously, and providing a pulsed white neutron source, with a neutron energy range between $1 \mathrm{meV}$ and $20 \mathrm{MeV}$, a time resolution of $1 \mathrm{~ns}$ and flight path lengths ranging from $10 \mathrm{~m}$ to $400 \mathrm{~m}$. The main effort of the experimental program at GELINA is devoted to applied research. Over the last decade, the IRMM has made an intense effort to improve the quality of neutron-induced cross section data in the resolved and unresolved resonance region. Due to the progress made, neutron-induced reaction cross section data, including full covariance information, can be obtained at GELINA from thermal up to the unresolved resonance region.
\end{abstract}

\section{Introduction}

The study of the resonance structure of cross sections requires high-resolution neutron energy dependent measurements. For measurement of cross section data in the resonance region an extremely good energy resolution is required to parameterize the cross sections in terms of resonance parameters. Experimentally, the resonance region is best studied at a pulsed white neutron source optimised for time-of-flight (TOF) measurements.

The TOF-facility GELINA has been especially designed for high-resolution cross section measurements in the resolved and unresolved resonance region [1]. It is a multi-user TOFfacility, providing a pulsed white neutron source, with a neutron energy range between $1 \mathrm{meV}$ and $20 \mathrm{MeV}$. The linear electron accelerator produces intense pulsed electron beams with a duration of $10 \mathrm{~ns}$ and a peak current of $10 \mathrm{~A}$, at a repetition rate which can range from $50 \mathrm{~Hz}$ to $800 \mathrm{~Hz}$. The electrons are accelerated to a maximum energy of $150 \mathrm{MeV}$. The $10 \mathrm{~ns}$ electron bunches are compressed to a duration of less than $1 \mathrm{~ns}$ by a post-acceleration compression magnet [2]. These high-energy electrons generate Bremsstrahlung $\gamma$-rays in a mercury cooled depleted uranium target, where neutrons are mainly produced by $(\gamma, \mathrm{n})$ and $(\gamma, \mathrm{F})$ reactions. At a $800 \mathrm{~Hz}$ operating frequency, the target delivers a neutron intensity of about $3.4 \times 10^{13}$ neutrons/s. To produce a significant number of neutrons in the energy below $100 \mathrm{keV}$, the fast neutrons produced in the uranium target are moderated by two waterfilled $\mathrm{Be}$ containers placed above and below the uranium target. Using suitable collimators, either the direct neutron spectrum with very good time resolution may be used, or the moderated neutron spectrum at reduced time resolution.

Time-of-flight measurements can be performed simultaneously at 10 flight-paths with lengths ranging from 10 to $400 \mathrm{~m}$. The measurement stations have special equipment, to perform

${ }^{a}$ Presenting author, e-mail: peter. schillebeeckx@eu.europa.ec transmission and partial (capture, fission, and inelastic) cross section measurements. Transmission measurements can be performed at a 25, 50, 100, 200 and $400 \mathrm{~m}$ flight path [3]. To study the Doppler broadening one of the transmission measurement stations is equipped with a cryostat, which is able to cool the samples down to $10 \mathrm{~K}$ [4]. Fission cross section measurements are performed at a 10 and $30 \mathrm{~m}$ station using Frisch gridded ionisation chambers and surface barrier detectors [5]. These measurement stations are also used to study (n,p) and (n, $\alpha)$ reactions. Inelastic scattering reactions are studied at a $30 \mathrm{~m}$ or $200 \mathrm{~m}$ station using HPGe-detectors [6]. Capture measurement systems are available at a 10, 30 and $60 \mathrm{~m} \mathrm{[7].}$

This contribution concentrates on developments related to capture measurements and to the analysis of total and capture cross section data in the resolved (RRR) and unresolved (URR) resonance region. For inelastic and fission cross section measurements we refer to contributions presented by C. Borcea [8] and C. Wagemans [9], respectively.

\section{Capture cross section measurements}

To study the neutron induced capture process, measurements can be performed at three stations with a nominal flight path length of 10, 30 and $60 \mathrm{~m}$. For applications such as the spin and parity assignment of resonances [10], the determination of partial cross sections and branching ratios [11] and isotope identification Ge-detectors are used at the $10 \mathrm{~m}$ station. To study the resonance structure of the capture cross sections hydrogen-free deuterated benzene $\left(\mathrm{C}_{6} \mathrm{D}_{6}\right)$ liquid scintillators are used applying the total energy detection principle in combination with the pulse height weighting technique [7]. In capture cross section measurements one observes the fraction of neutrons that interact in the sample and create a signal in the detection system. This fraction, i.e. the experimental yield $\mathrm{Y}_{\text {exp }}$, is obtained from the ratio of the counts seen by 


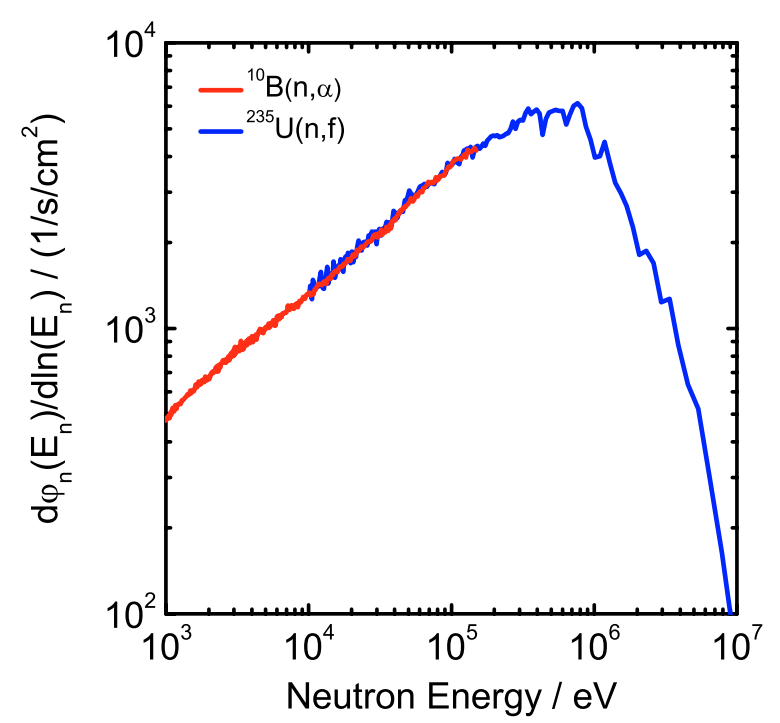

Fig. 1. The neutron spectrum at the $60 \mathrm{~m}$ capture station with the accelerator operating at $800 \mathrm{~Hz}$ and a 0.042 atoms/barn thick ${ }^{10} \mathrm{~B}$ overlap filter.

the capture detector to the incident neutron flux $\varphi_{\mathrm{n}}$ and is expressed as a function of the total, capture and scattering cross section. The parameterisation of the response of the capture detector in terms of resonance parameters requires the knowledge of the shape of the neutron flux, the resolution of the TOF-spectrometer and the detection efficiency and neutron sensitivity of the capture detection system.

\subsection{Neutron flux and resolution}

A study of both the moderated and direct neutron spectrum of GELINA obtained from electron-photon-neutron coupled Monte Carlo simulations can be found in ref. [12]. At the capture measurement stations the shape of the neutron flux is simultaneously measured using either a ${ }^{10} \mathrm{~B}$ or a ${ }^{235} \mathrm{U}$ ionization chamber placed in the neutron beam $1 \mathrm{~m}$ closer to the neutron source than the capture sample. The ${ }^{10} \mathrm{~B}(\mathrm{n}, \alpha)$ reaction is used for measurements below $150 \mathrm{keV}$ and the ${ }^{235} \mathrm{U}(\mathrm{n}, \mathrm{f})$ reaction for energies above $150 \mathrm{keV}$. The neutron spectrum at the $60 \mathrm{~m}$ capture station, obtained from the ${ }^{10} \mathrm{~B}(\mathrm{n}, \alpha)$ and ${ }^{235} \mathrm{U}(\mathrm{n}, \mathrm{f})$ measurements, with a 0.042 atoms/barn thick ${ }^{10} \mathrm{~B}$ overlap filter and the accelerator operating at $800 \mathrm{~Hz}$, is shown in figure 1.

To extract resonance parameters from a resonance shape analysis the knowledge of the resolution function is required. A description of the various components of the resolution function of a TOF-facility is given in ref. [13]. A detailed study of the resolution function of GELINA based on Monte Carlo simulations, which were benchmarked with experimental data, can be found in refs. [12,14].

\subsection{Weighting function for $C_{6} D_{6}$ detectors}

Tain et al. [15] have already demonstrated that accurate weighting functions can be obtained from Monte Carlo simulations provided that the geometry description reflects the experimental conditions and that the $\gamma$-ray transport in the sample and the detector environment is accounted for. In ref. [7] we have shown that due to the finite sample size the weighting function depends on the resonance strength and may differ from resonance to resonance. For weak resonances a homogeneous distribution of the $\gamma$-rays is valid, while for strong resonances one needs to account for the flux attenuation. Hence, each resonance requires in principle its own weighting function. This is from a practical point of view not realistic. We proposed in ref. [7] a practical solution which accounts for both the influence of the resonance strength and the neutron sensitivity of the detection system: the experimental yield $\mathrm{Y}_{\exp }$ is deduced using a weighting function calculated for $\gamma$-rays which are homogeneously distributed in the sample and a correction factor $\left(\mathrm{K}_{\mathrm{c}}\right)$ is applied when the experimental yield is expressed as a function of the theoretical yield. This theoretical yield is a sum of the yield due to neutrons which are captured in the sample $\left(\mathrm{Y}_{\mathrm{c}}\right)$ and to neutrons which are scattered in the sample and create a detectable signal due to a subsequent capture reaction in the detector and its environment $\left(\mathrm{Y}_{\mathrm{n}}\right)$. The latter contribution depends on the socalled neutron sensitivity of the detection system. Since the neutron sensitivity does not depend on the resonance strength one can not correct the experimental yield and the correction factor $K_{c}$ needs to be applied on the calculated capture yield $\left(\mathrm{Y}_{\mathrm{c}}\right)$.

The contribution due to scattered neutrons creates a time dependent prompt background which cannot be distinguished from the real capture events and therefore contributes to the experimental yield. The sensitivity to neutrons of the capture detection systems used at GELINA were also calculated by the Monte Carlo simulations and validated with experimental data [7]. We have demonstrated in ref. [7] that the correction of the neutron sensitivity needs to be included in the resonance shape analysis. Not all resonance shape analysis codes include a direct correction for the neutron sensitivity in the description of the experimental yield as proposed in ref. [7]. In such case a correction is applied to the observed radiation width which is deduced from an analysis without accounting for the neutron sensitivity. In ref. [7] it is shown that such a correction for the neutron sensitivity results in a systematic underestimation of the radiation width.

\section{Data reduction and analysis}

\subsection{Data reduction with AGS}

As a response to the increasing importance and need of full covariance information, the IRMM and CEA Saclay started a collaboration to develop a full tested and documented version of the Analysis of Generic TOF_Spectra (AGS) package [16]. The AGS system, consisting of a set of stand-alone C programs, has been developed to transform count rate spectra in an efficient and convenient way into a final observable, transmission factor or partial reaction yield, which can be used for model fitting. The package performs a full propagation of uncertainties, starting from the uncorrelated uncertainties 
due to counting statistics. The final output includes a complete covariance matrix accounting for both correlated and uncorrelated uncertainty components. In the AGS system the covariance matrix is split in two parts, separating the uncorrelated and correlated components. The uncorrelated part can be represented as a diagonal matrix, while the correlated part may be expressed as the product of a rectangular matrix with its own transpose.

The AGS data structure results in a substantial reduction of space for data storage and provides a convenient input structure suitable for subsequent data analysis involving nuclear reaction models. In addition, the structure allows a full documentation of the propagation of uncertainties and the impact of the various sources of uncertainties can be verified through each step of the reduction process. Reference [17] gives a detailed discussion on the correlated and uncorrelated uncertainty components of the ${ }^{232} \mathrm{Th}(\mathrm{n}, \gamma)$ capture cross section data in the unresolved resonance region based on the AGS output.

\subsection{Parameterisation of cross section data}

For the analysis of transmission and capture data in the RRR obtained at GELINA the REFIT [18] code is mostly used. The code is based on the Reich-Moore approximation of the R-matrix theory and accounts for self-shielding, multiple scattering and Doppler effects, and the resolution of the TOFspectrometer. We initiated a collaboration with Serco Ltd. and the University of Birmingham to produce a tested and documented version of the current version of REFIT. A proposal has been submitted to the EFNUDAT project [19] to support the implementation of recent improvements for the analysis of capture and transmission data. The improvements relate to:

- The analysis procedure of capture data proposed in ref. [7];

- The treatment of variation in sample thickness for the analysis of transmission data [4];

- The description of the resolution function by Monte Carlo simulation and analytical expression in ref. [20].

For the analysis in the URR a procedure has been developed by Sirakov et al. [21] which is predominantly based on the approach implemented in FITACS [22]. A link to the optical model is used for information about the energy dependence of the potential scattering radius and the neutron strength functions. Depending on the quality of the available experimental data, this link can serve as a constraint in the optimisation of the parameters. The average cross sections are expressed in terms of transmission coefficients by applying the Hauser-Feshbach statistical reaction theory including widthfluctuations. Both an ENDF-6 oriented approach and a more rigorous scheme, not restricted by format limitations, are considered.

\section{Applications}

The development and improvement of a comprehensive cross section database is essential for many areas of research and technology. For nuclear power production, neutron-induced reactions are definitely the most important interactions. Accurate neutron cross sections play a crucial role not only for nuclear power, but also in many other disciplines such as astrophysics, medicine, security and archaeology.

\section{1 ${ }^{206} \mathrm{~Pb}$ (n,tot) and $(\mathrm{n}, \gamma)$ measurements}

Neutron total and capture reaction cross section measurements for ${ }^{206} \mathrm{~Pb}$ have been performed at GELINA in the energy range from 1 to $620 \mathrm{keV}$ [23]. For the capture measurements special experimental conditions and data analysis procedures were implemented to reduce systematic bias effects as much as possible and to avoid correction factors requiring knowledge about the $\gamma$-ray emission cascade. Due to these precautions the correlated uncertainty component on the capture data was reduced to $2 \%$. From the capture data, accurate total radiation widths were extracted.

In the region up to $620 \mathrm{keV}, 304$ resonances were observed and analyzed, compared to only 234 resonance in previous transmission experiments [24] and 221 listed in ENDF/BVI.8. The final resonance parameters, deduced from an analysis with REFIT, together with the experimental capture yield and transmission factors are listed in the EXFOR data library and the capture areas are included in the latest compilation of Mughabghab. From the capture data photon strength functions have been deduced which do not show an enhancement of M1 transition and do not confirm the existence of a doorway state in the photon channel.

Figure 2 shows a comparison between the experimental yield with the calculated yield using the parameters obtained from the GELINA data and the ENDF/B-VI.8 resonance parameters, which are also used for the latest ENDF/B-VII.0 version. Due to the lack of capture cross section data above $200 \mathrm{keV}$, significant discrepancies in the radiation widths are observed.

From the experimental observed capture areas up to $620 \mathrm{keV}$ the Maxwellian Averaged Capture (MAC) cross sections as function of temperature were derived with an accuracy better than the required 5\%. The MACs obtained in this work are systematically lower than the values compiled by Bao et al. [25] and higher than the ones deduced from cross section data in the evaluated data libraries. It was shown that the resonances, which were observed from measurements at GELINA and not reported in ENDF/B-VI.8, contribute for more than $8 \%$ to the MAC cross sections for energies above
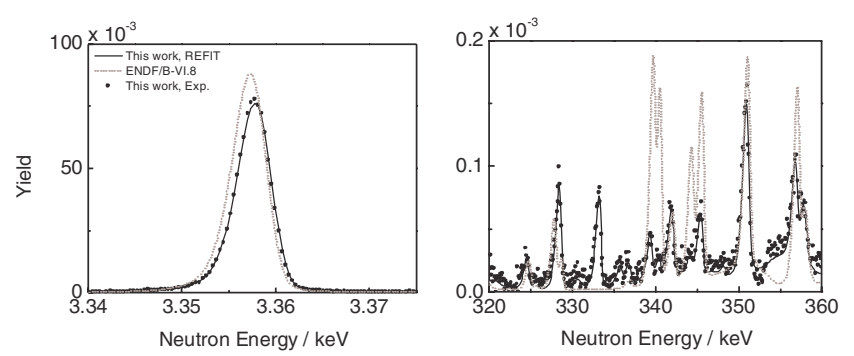

Fig. 2. The experimental yield together with the theoretical yield deduced from an analysis with REFIT and the ENDF/B-VI.8 parameters. 
$\mathrm{kT}=30 \mathrm{keV}$. The results in ref. [23] demonstrate that the abundances deduced from resonance data that do not include the capture areas which were deduced at GELINA but not included in the evaluated data files are overestimated by at least $8 \%$.

\subsection{Cross section data for stable fission products}

Improved capture cross sections for various stable fission products are important to extend and optimize the fuel cycle associated with present nuclear power plants and for criticality safety of spent fuel storage and transportation of spent fuel in licensed shipping casks. To improve these data, the IRMM started in collaboration with ORNL (US) measurements at GELINA for ${ }^{103} \mathrm{Rh},{ }^{133} \mathrm{Cs},{ }^{155} \mathrm{Gd}$ and ${ }^{55} \mathrm{Mn}$. For more details on the progress made for these applications we refer to the contribution of Mihailescu et al. [26].

\subsection{Evaluation of ${ }^{232} \mathrm{Th}$ total and partial cross section data in the URR}

As a part of the Coordinated Research Project "Evaluated Nuclear Data for the Th-U Fuel Cycle" organized by the International Nuclear Data Committee of the IAEA, the ${ }^{232} \mathrm{Th}$ total and partial cross sections has been evaluated in the energy range from 4 to $100 \mathrm{keV}$. The ENDF-6 oriented evaluation scheme with a link to the optical model, as proposed by Sirakov et al. [21], has been applied. The evaluation resulted from a simultaneous analysis of transmission and capture measurements, including the most recent capture data obtained from measurements at the time of flight facilities GELINA at Geel (B) and n-TOF at CERN (CH). The evaluation has been verified by a comparison with results from self-indication measurements and a full covariance matrix was generated.

\subsection{NUDAME}

Several experiments carried out at GELINA are supported by the Transnational Access facility Nuclear Data Measurements NUDAME of the European Commission [27]. The following experiments have already been performed at GELINA in the framework of NUDAME:

- High-resolution capture and transmission measurements of ${ }^{\text {nat }} \mathrm{Hf}$ (CEA Cadarache),

- Capture and transmission measurements on ${ }^{\text {nat }} \mathrm{Cd}$ (IAEA Vienna),

- Test of data acquisition with $\mathrm{C}_{6} \mathrm{D}_{6}$ detectors using fast signal digitizers (INFN Bari).

\subsection{Ancient Charm}

Two measurement stations (at 10 and $30 \mathrm{~m}$ ) are used to determine the elemental composition of materials and objects by "Neutron Resonance Capture Analysis" (NRCA). This technique has been developed at the IRMM in collaboration with the Delft University of Technology. The method is based on the use of neutron resonances as fingerprints to identify and quantify elements [28]. Due to the expertise at the IRMM in this field $[28,29]$, the GELINA facility is used to contribute to the Ancient Charm project [30], which is financed by the European Commission "New and Emerging Science and Technology" programme. The project's title is an acronym for "Analysis by Neutron resonant Capture Imaging and other Emerging Neutron Techniques: new Cultural Heritage and Archaeological Research Methods". Aim of the project is to develop NRCA and the other neutron-based analysis techniques into non-invasive methods for imaging of the elemental and phase composition of cultural heritage objects. The interest for such an analysis will be investigated with a broad theoretical scope to provide the cultural heritage foundations of Ancient Charm.

This work has been performed with financial support by the European Commission within the "New and Emerging Science and Technology" programme under contract No. 15311.

\section{References}

1. A. Bensussan, J.M. Salome, Nucl. Instrum. Meth. 155, 11 (155).

2. J.M. Salome, R. Cools, Nucl. Instrum. Meth. 179, 13 (1981).

3. S. Kopecky, A. Brusegan, Nucl. Phys. A 773, 173 (2006).

4. S. Kopecky et al. (these proceedings AID 391).

5. J. Heyse et al., Nucl. Sci. Eng. 156, 211 (2007).

6. L.C. Mihailescu et al., Nucl. Phys. A 786, 1 (2007).

7. A. Borella et al., Nucl. Instrum. Meth. A (accepted).

8. C. Borcea et al. (these proceedings AID 329).

9. C. Wagemans et al. (these proceedings AID 113).

10. L. Zanini et al., Phys. Rev. C 61, 054616 (2000).

11. A. Borella et al. (these proceedings AID 431).

12. M. Flaska et al., Nucl. Instrum. Meth. A 531, 394 (2004).

13. A. Brusegan et al., J. Nucl. Sci. Technol. Suppl. 2, 685 (2002).

14. D. Ene et al. (these proceedings AID 330).

15. J. Tain et al., J. Nucl. Sci. Technol. Suppl. 2, 689 (2002).

16. C. Bastian et al., Physor-2006, ANS Topical Meeting on Reactor Physics, Vancouver, BC, Canada, 10-14 September 2006.

17. A. Borella et al., Nucl. Sci. Eng. 152, 1 (2006).

18. M.C. Moxon, J.B. Brisland, AEA-INTEC-0630, AEA Technology, October 1991.

19. http://www.efnudat.eu/.

20. Kopecky et al., in WONDER 2006, Cadarache, October 2006.

21. I. Sirakov et al., in WONDER 2006, Cadarache, October 2006.

22. F.H. Fröhner, Nucl. Sci. Eng. 103, 119 (1989).

23. A. Borella et al., Phys. Rev. C (to be published).

24. D.J. Horen et al., Phys. Rev. C 20, 478 (1979).

25. Z.Y. Bao et al., At. Data Nucl. Data Tables 76, 70 (2000).

26. L.C. Mihailescu et al. (these proceedings AID 617).

27. http://www.irmm.jrc.be/html/nudame/.

28. H. Postma, P. Schillebeeckx, J. Radioanal. Nucl. Chem. 265, 297 (2005).

29. G. Noguere et al., Nucl. Instrum. Meth. A (accepted).

30. G. Gorini and the Ancient Charm collaboration, Nuovo Cimento C 30, 47 (2007). 